\title{
Selection Rules for the Vibrational Spectra of High Polymers
}

\author{
C. Y. $\operatorname{LIANG}^{1}$ \\ The Harrison M. Randall Laboratory of Physics, University of \\ Michigan, Ann Arbor, Michigan
}

\begin{abstract}
With the aid of the Born eyclic postulate, the selection rules for the vibrational modes of a crystalline polymer and an oriented infinite chain-molecule have been discussed. The necessary conditions for the modes to be active in the infrared or Raman spectrum have been deduced from the translation group of the crystal or of the chain. Approximate methods lave also been discussed for a finite chain-molecule and for a high polymer of amorphous form.
\end{abstract}

\section{INTRODUCTION}

It is well known that the methods of group theory can be used to analyze the spectra of small molecules and crystals. Some authors have also applied this theory to the spectra of high polymers $(1-6)$. One difficulty in applying group theory to the spectra of high polymers is that the polymer samples available are always partly crystalline and partly amorphous, and mixed spectra are often observed. In addition, the interpretation of the spectra in the overtone region is generally complicated.

In the present work we will discuss the necessary conditions for the vibrational modes of crystalline or oriented polymers to be active in the infrared or Raman spectrum. Approximate methods will also be discussed for finite chain molecules and for high polymers of amorphous form.

\section{THE SELECTION RULES FOR THE SPECTRA OF CRYSTALLINE POLYMERS}

Let us consider first the spectra of high polymers in the crystalline state. In this case we can treat the problem the same as that of an ordinary three dimensional crystal. The basic postulate for the possible normal vibrations of a threedimensional lattice is the Born cyclic lattice condition (7). From Born dynamics the normal frequencies are functions of the wave-vector $\mathbf{k}$. Let us consider a block in the infinite crystal. The cyclic condition requires

$$
\mathbf{k}_{\alpha \beta \gamma}=\frac{\alpha}{n_{1}} \mathbf{b}_{1}+\frac{\beta}{n_{2}} \mathbf{b}_{2}+\frac{\gamma}{n_{3}} \mathbf{b}_{3}
$$

${ }^{1}$ Present address: Research and Development Division, American Viscose Corporation, Marcus Hook, Pennsylvania. 
where $\alpha, \beta$, and $\gamma$ are integers from 0 to $\left(n_{1}-1\right),\left(n_{2}-1\right)$ and $\left(n_{3}-1\right)$, respectively. The $n_{1}, n_{2}$, and $n_{3}$ are the number of lattice vectors $\mathbf{a}_{1}, \mathbf{a}_{2}$, and $\mathbf{a}_{3}$, respectively, counted along the principle axes. The $b_{i}$ 's are the reciprocal vectors. The scalar product $\mathbf{a}_{i}, \mathbf{b}_{j}$ is one for $i=j$ and zero for $i \neq j$. The physical interpretation of the cyclic condition is that any vibrational mode of the atoms in the block must repeat again and again inside the crystal. In other words, all equivalent blocks of the crystal are oscillating in phase. In applying this postulate, an immediate question arises; namely, how to select this block from the crystal. There are a few cases that can be mentioned: (a) In the original Born dynamics, the selected block containes $n^{3}$ unit cells with $n$ any larger integer. (b) In the finite space group discussed by Winston and Harford (8), the block contains $n_{1} n_{2} n_{3}$ unit cells with $n_{1}, n_{2}, n_{3}$ integers. (c) In the special case of Born dynamics used by Raman in interpreting the spectrum of Diamond (9), the block contains $2^{3}$ or 8 unit cells. (d) In the factor group of the space group discussed by Bhagavantam and Venkatarayudu (10) [or the unit cell group by Hornig (11)], the block contains only one unit cell.

For the selection rules concerned only with the possible active fundamentals of a crystal, the analysis based on the factor group (unit cell group) of the space group has been proven correct. Only the normal vibrations deduced from the factor group may be active in the infrared or Raman spectrum. These modes are all totally symmetrical to the lattice translation group; or in other words, all the unit cells swing in phase. This is the necessary condition for the modes to be active and can be shown as follows. Consider a block of the crystal with $n_{1}$ $n_{2} n_{3}$ unit cells. The symmetry operations of the translation group are

$$
\mathbf{t}_{\lambda \mu \nu}=\lambda \mathbf{a}_{1}+\mu \mathrm{a}_{2}+\nu \mathbf{a}_{3},
$$

where $\lambda=0,1,2, \cdots, n_{1}-1$, etc. The irreducible representations of the translation group (12) are given in Table I. By the well-known formulas of group theory, we can calculate the number of active and forbidden modes under the translation group. These are also given in Table I. Only the modes under the totally symmetrical representation $\Gamma(0)$ are active. If the unit cell group is for example, $C_{1}, C_{s}$ or $C_{2}$, the condition for the modes to be active is necessary and sufficient. If the unit cell group is of higher symmetry, then all the modes which are totally symmetrical to the translation group may not be active, and the condition becomes necessary but not sufficient. The more complete selection rule in this case can be deduced by considering the finite space group of a chosen block of the crystal.

For overtones and combinations of the spectra of crystals we have to consider the block of $n_{1} n_{2} n_{3}$ unit cells with sufficiently large numbers of $n_{i}$ 's. Since numerous overtones and combinations of inactive fundamentals may be active, the bands are thus overlapped with each other. The necessary condition for the 


\section{TABLE I}

Selection Rules for the Normal Vibrational Modes of Crystals Under the Lattice Transhation Group (A-Active, F-Forbidden)

\begin{tabular}{|c|c|c|c|c|c|c|c|c|}
\hline \multicolumn{2}{|c|}{ E } & $\mathbf{t}_{160}$ & $\cdots$ & $t_{\lambda \mu \nu}$ & $\cdots$ & $\mathbf{N}_{\mathbf{i}}$ & I. $\mathbf{R}$. & Raman \\
\hline$\Gamma(0)$ & 1 & 1 & $\cdots$ & 1 & $\ldots$ & $3 s$ & A & A \\
\hline$\Gamma\left(\mathbf{k}_{100}\right)$ & 1 & $\exp \left(2 \pi i \mathbf{k}_{100} \cdot \mathbf{t}_{100}\right)$ & $\cdots$ & $\exp \left(2 \pi i \mathbf{k}_{10 D} \cdot \mathbf{t}_{\lambda \mu \nu}\right)$ & $\cdots$ & $3 s$ & $\mathrm{~F}$ & $\mathrm{~F}$ \\
\hline$\Gamma\left(\mathbf{k}_{\alpha \beta_{\gamma}}\right)$ & 1 & $\exp \left(2 \pi i \mathbf{k}_{\alpha \beta \gamma} \cdot \mathbf{t}_{100}\right)$ & $\cdots$ & $\exp \left(2 \pi i \mathbf{k}_{\alpha \beta \gamma} \cdot \mathbf{t}_{\lambda \mu \nu}\right)$ & $\cdots$ & $3 s$ & $F$ & $F$ \\
\hline$U(R)$ & $n_{1} n_{2} n_{3} s$ & 0 & $\ldots$ & 0 & $\ldots$ & & & \\
\hline$X^{v}$ & 3 & 3 & $\ldots$ & 3 & $\ldots$ & & & \\
\hline$X^{\text {s.t. }}$ & 6 & 6 & $\ldots$ & 6 & $\ldots$ & & & \\
\hline
\end{tabular}

$s=$ number of atoms in the unit cell. $U(R)=$ number of invariant atoms in the block under the symmetry operation $R . X^{\mathrm{v}}=$ character of the vector representation $= \pm 1+2$ $\cos \phi . X^{\text {s.t. }}=$ character of the symmetric tensor representation $=2 \cos \phi \cdot X^{\mathrm{v}}, N_{i}=$ total number of normal modes under $i$ th irreducible representation.

overtone or combination to be active in the infrared or Raman spectrum is that the combined representations must be totally symmetrical to the translation group. Consider the combination $\Gamma\left(\mathbf{k}_{i}\right)$ and $\Gamma\left(\mathbf{k}_{f}\right)$. From Table I we obtain the representation of $\Gamma^{*}\left(\mathbf{k}_{i}\right) \cdot \Gamma\left(\mathbf{k}_{f}\right)$ as

$$
1, \cdots, \exp \left[2 \pi i\left(\mathbf{k}_{f}-\mathbf{k}_{i}\right) \cdot t_{\lambda \mu \nu}\right], \cdots, \cdots .
$$

if $\mathbf{k}_{f}=\mathbf{k}_{i}$, then $\Gamma^{*}\left(\mathbf{k}_{i}\right) \cdot \Gamma\left(\mathbf{k}_{f}\right)=\Gamma(0)$, which is the totally symmetric representation of the translation group. Hence, this combination may be active in the infrared or Raman spectrum. If $\mathbf{k}_{\boldsymbol{f}} \neq \mathbf{k}_{i}$, the combination is inactive.

Let us consider next the possibility of using the site group (13) to analyze the spectra of crystalline high polymers. If the bands due to the interaction of neighboring groups in the same unit cell are not resolved, the site group could be used as an approximation. For example, crystalline polyisobutylene $\left[-\mathrm{CH}_{2} \mathrm{C}\left(\mathrm{CH}_{3}\right)_{2}-\right]_{n}$ may have a orthorhombic unit cell (14). The polymer has a spiral chain with 8 monomer units in a period. There are two chains passing through the unit cell. The space group of this structure is $D_{2}{ }^{4}$, and its factor group is isomorphic to the point group $D_{2}$. The total number of normal modes calculated from the factor group is $144\left(A_{1}+B_{1}+B_{2}+B_{3}\right)$. Since the modes of species $B_{1}, B_{2}$, and $B_{3}$ are infrared active, we may expect to observe $(3 \times$ 144) $-3=429$ bands. Actually, we have observed less than 20 distinct bands in the fundamental region. In this case we need not use the analysis based on the unit cell group. We could interpret the spectrum by considering only one monomer unit. 
It may be noted that the polarization properties of the spectra of crystals cannot be predicted from the site group. This is a great disadvantage of this approximation. In addition, the overtones and combinations of the fundamentals deduced from the unit cell group cannot be interpreted. For example, since the unit cell group of crystalline polyethylene $(1,4)$ contains the symmetry operation of center of inversion, no overtone or combination of the infrared fundamentals can be active in the infrared. If the analysis is based on the local symmetry $C_{2 v}$, all the overtones of the infrared fundamentals are active in the infrared. The approximation is thus in contradiction to the more accurate approach.

\section{THE SPECTRA OF ONE-DIMENSIONAL ORIENTED HIGH POLYMERS}

If the interaction between chains in the same unit cell is weak, we can set up the selection rules for the spectra of oriented long chain polymers by considering the one-dimensional space group. The treatment for the one-dimensional lattice can be deduced from the three-dimensional case discussed in the last section. However, there are two main differences:

(1) For the three-dimensional crystal, the symmetry axes, $C_{p}$, are restricted to $p=1,2,3,4$, and $6(15)$. For the one-dimensional case, $p$ may be any real number. Let us, for example, consider a spiral chain. If $p$ is a whole number $N$, (for example, $p=8$ for polyisobutylene), the chain will repeat after $N$ monomer units. If $p$ is a whole number $N$ plus a real fraction $h / g$, then the chain will repeat after $\mathrm{Ng}+h$ monomer units. If $p$ is an irrational number, the chain will never repeat and the cyclic group $C_{p}$ becomes infinite. These cases are all possible.

(2) There are no pure rotational modes in the three-dimensional case. In the one-dimensional case one pure rotational mode, the rotation about the chain axis, is present. If $s$ is the number of atoms per unit cell, then $3 s-3$ is the number of nonzero fundamental frequencies under the unit cell group in the three-dimensional case. In the one-dimensional case the number of nonzero fundamental frequencies under the factor group of the one-dimensional space group is $3 s^{\prime}-4$, where $s^{\prime}$ is the number of atoms per repeating unit of the chain.

It may be pointed out that the one-dimensional approximation does not correspond to the analysis of the site group. The site group is a point group, while the one-dimensional space group is not. Furthermore, the site group is a subgroup of the unit cell group, but the factor group of the one-dimensional space group is not in general a sub-group of the unit cell group. For example, the unit cell group of erystalline polyisobutylene, as we have mentioned, is isomorphic to $\mathrm{D}_{2}$. For a single spiral chain of polyisobutylene the factor group of the onedimensional space group is isomorphie to $\mathrm{C}_{8}$, which is not a subgroup of $\mathrm{D}_{2}$. In the case of crystalline rubber hydrochloride (16) the factor group of one- 
dimensional space group for a single chain is isomorphic to $\mathrm{C}_{\mathbf{s}}$, which is a subgroup of the unit cell group $C_{2 h}$.

Next let us determine the necessary conditions for the modes to be active in the one-dimensional case. Let $n$ be the number of the smallest repeating unit in a selected segment of the infinite chain. Then, by analogy to the Born cyclic condition, we have

$$
\mathbf{k}_{l}=\frac{l}{n} \mathbf{b},
$$

where $l=0,1,2, \cdots, n-1$. The physical interpretation is that for the permitted modes all equivalent segments of the chain oscillating in phase. If we take $n=1$, the treatment is reduced to the analysis of the factor group of the one-dimensional space group. Just as in the three-dimensional case, the necessary condition for the fundamentals to be active in the infrared or Raman spectrum is that the smallest repeating units of the chain must all swing in phase. This condition can be formulated by the phase difference $\theta$ between adjacent characteristic units which can be reached from each other by screw rotations of the chain. Let $p$ be the number of characteristic units in a repeating period. It can be seen that if something occurred at the first characteristic unit of the period, the same thing occurred at the first characteristic unit of the next period, and so on. Therefore, $p \theta$ must be $2 \pi$ times a whole number. We then have

$$
\theta=(2 \pi / p) \mathrm{r}, \text { with } r=0,1,2, \cdots, p-1 .
$$

For $r>p-1$ we get nothing new since $r=p$ is equivalent to $r=0$, and so on. Since $-r$ is equivalent to $p-r$, we can also put the condition in the following form:

$$
\theta=(2 \pi / p) r
$$

with $r=0, \pm 1, \pm 2, \cdots, \pm(p-1) / 2$ for $p$ odd, or $r=0, \pm 1, \pm 2, \cdots$, $\pm(p-2) / 2, p / 2$ for $p$ even. An infinite planar zig-zag chain of $\left(-\mathrm{CH}_{2} \mathrm{CH}_{2}-\right)_{\infty}$ is a special example with $p=2$, and $\theta=0$ and $\pi$. For a single chain of polychlorotrifluoroethylene (5), we have $p=14$, and $\theta=(2 \pi / 14)$, with $r=0$, $\pm 1, \cdots, \pm 6$ and 7 .

So far we have discussed the necessary conditions for the vibrational fundamental modes of an infinite chain polymer to be active in the infrared and Raman Spectrum. For the overtones and combinations, we have to consider, as in the three-dimensional case, a large segment of the chain, and treat the problem with a finite one-dimensional space group. Let us consider a segment of the plane zig-zag chain of $\left(-\mathrm{CH}_{2} \mathrm{CH}_{2}-\right)_{n}$. We can treat the problem by the factor group of the one dimensional space group by putting $n=1$. This factor group is isomorphic to $D_{2 h}$ with eight symmetry operations $(1,4)$. If we choose $n=2$, 
the one dimensional finite space group contains 16 symmetry operations, which may be easily shown to form a non-Abelian group. Degenerate modes are thus present, because the irreducible representations of a non-Abelian group cannot all be one-dimensional. It is interesting to note that, for a point group, if the highest symmetry axis is two fold, we can have only nondegenerate modes. This conclusion is no longer true in general for a finite space group.

\section{THE SPECTRA OF FINITE CHAINS}

In the foregoing discussion we have used the Born cyclic postulate to set up the selection rules for both the three dimensional crystal and the infinite chain polymer. For a finite chain the situation is different. Rigorous selection rules in such a case may be deduced by considering the point symmetry of the whole molecule. Let us consider, for example, the normal paraffins $C_{N} \mathrm{H}_{2 N+2}(N>2)$ with plane zig-zag backbones. The methyl groups at the ends may be arranged either in trans or in cis form. The cis form for odd $N$ belongs to the point symmetry $C_{2 v}$. Both the trans form for odd $N$ and the cis form for even $N$ belong to the point symmetry $C_{s}$. The remaining case the trans form with even $N$, belongs to the point symmetry $C_{2 h}$. The selection rules for all these cases can be worked out readily. If the chain is quite long and extended (plane zig-zag form or repeating helix), we can treat the problem by the following approximate method. Consider one smallest repeating unit of the chain at a certain normal vibration. By various phase combinations of the vibrations between successive units, we can obtain a series of normal vibrations of the chain. If all the units of the chain swing in phase, the mode is potentially active. The intensity $\left(I_{s}\right)$ of the potentially active spectral line is proportional to $(m A)^{2}$, where $m$ is the number of the repeating units in the chain and $A$ is the maximum amplitude of the dipole moment change in the unit. If, for the same type of normal vibrations in the unit, the units of the chain do not all swing in phase, the modes may still be active, but the intensities are much weaker. The condition for the modes to be weakly active is that an odd integer of half a wavelength (the wavelength of the normal pattern) must be completed within the chain. The intensity $\left(I_{w}\right)$ of the weakly active spectral is approximately proportional to

$$
\left(\frac{1}{2} \cdot \frac{m A}{2 q+1}\right)^{2}
$$

where $q=0,1,2, \cdots$. Hence, we have

$$
I_{w} / I_{s}=\frac{1}{[2(2 q+1)]^{2}}
$$

The potentially active mode and the weakly active modes of the same type of vibration form a branch of frequencies or a band. 


\section{THE SPECTRA OF AMORPHOUS POLYMERS}

For the spectra of high polymers in the amorphous state, no exact selection rules are possible. The spectrum of the amorphous form is generally more diffuse than that of the crystalline form. A number of other differences between the spectra of these two forms has been observed. For examples, the strong bands of the infrared spectrum of polethylene are doublets in the crystalline form but become singlets in the amorphous form (4). In the spectrum of polethylene terephthalate the relative intensity of the band at $972 \mathrm{~cm}^{-1}$ is increased markedly when the crystallinity of the sample is increased (17). For the infrared spectrum of rubber $(18,19)$ the differences are: (a) The band at $1665 \mathrm{~cm}^{-1}$ is nearly the same for both crystalline and amorphous forms. (b) The bands at 836, 1135, 1330, and $1365 \mathrm{~cm}^{-1}$ change their intensities and positions with change of form. (c) The bands at 870 and $962 \mathrm{~cm}^{-1}$ are observed only in crystalline rubber. (d) The band $1240 \mathrm{~cm}^{-1}$ of amorphous rubber is resolved into three components at 1245,1230 , and $1210 \mathrm{~cm}^{-1}$ in the crystalline form. It may be that the band observed only in the crystalline state is due to the interaction between neighboring chains in the unit cell $(3,4,20)$, while the band observed only in the amorphous state may be due either to breakdown of selection rules operative in the crystal or to the existence of different rotational isomers. In interpreting the spectra of amorphous high polymers, we have to consider all the characteristic groups of atoms in the polymer. If a special arrangement of a characteristic group of atoms is considered, local symmetry can be used. For example, the selection rules for the normal vibrations associated with the benzene ring in amorphous polystyrene may be deduced from the local symmetry $C_{2 v}$ of the ring (21).

\section{ACKNOWLEDGMENT}

The author wishes to thank his wife and Professor G. B. B. M. Sutherland for their very helpful discussions, and Dr. Rhoda J. Buser, Dr. Julian H. Gibbs, and Dr. E. W. Lothrop for their review of this paper and their many helpful suggestions.

ReCeiveis: February 25, 1957

\section{REFERENCES}

1. P. W. Higgs, Proc. Roy. Soc. A220, 472 (1953).

2. M. C. Toвin, J. Chem. Phys. 23, 891 (1955).

3. C. Y. Liang, S. Krimm, and G. B. B. M. Sutherland, J. Chem. Phys. 25, 543 (1956).

4. S. Krimm, C. Y. Liang, and G. B. B. M. Sutherland, J. Chem. Phys. 25, 549 (1956).

5. C. Y. Liang ANd S. Krimm, J. Chem. Phys. 25, 563 (1956).

6. S. Krimm and C. Y. Liang, J. Polymer Sci. 22, 95 (1956).

7. H. M. J. Sмгтн, Phil. Trans. Roy. Soc. A241, 105 (1948); see also refs. 1-6.

8. H. Winston and R. S. Halford, J. Chem. Phys. 17, 607 (1949).

9. C. V. Raman, Proc. Indian Acad. Sei. 18A, 237 (1943).

10. S. Bhagavantam and T. Venkatarayudu, Proc. Indian Acad. Sci. 9A, 224 (1939).

11. F. Hornig, J. Chem. Phys, 16, 1063 (1948). 
12. F. Seitz, Ann. Math. 37, 17 (1936).

13. R. S. HALFORD, J. Chem. Phys. 14, 8 (1946).

14. C. S. Fuller, C. J. Frosch, and N. R. Pape, J. Am. Chem. Soc. 62, 1905 (1940).

15. See for example, W. H. Zachariasen, "Theory of X-Ray Diffraction in Crystals,"' p. 31.

Wiley, New York, 1951.

16. C. W. Bunn and E. V. Garner, Proc. Roy. Soc. A189, 39 (1947).

17. W. H. CobBs, JR., AND R. L. Burton, J. Polymer Sci. 10, 275 (1953).

18. G. B. B. M. Sutherland and A. Vallance Jones, Discussions Faraday Soc. 9, 281 (1950).

19. R. A. Saunders and D. C. Smith, J. Appl. Phys. 20, 953 (1949).

20. R. S. Strin, J. Chem. Phys. 23, 734 (1955).

21. C. Y. Liang ANd S. Krimm, J. Polymer Sci. in press (1957). 